\title{
Escalas de Estilos Interpessoais: evidências de validade convergente
}

\author{
Mauro de Oliveira Magalhães ${ }^{1}$ \\ Universidade Federal da Bahia, Salvador-BA, Brasil
}

\section{RESUMO}

O estilo interpessoal é um construto multidimensional definido como a maneira que o indivíduo busca por aceitação e status social. Esta pesquisa deu continuidade ao estudo das evidências de validade do Inventário Escalas de Estilos Interpessoais (ESEI) por meio da correlação com outras medidas de personalidade. Participaram da investigação 848 pessoas com idades entre 18 e 60 anos $(M=28,50)$, 328 homens e 520 mulheres, residentes nas Regiões Sul e Nordeste, que responderam, presencialmente ou mediante plataforma on-line (http://www.surveymonkey.com), ao ESEI, a uma medida dos cinco grandes fatores de personalidade (International Personality Item Pool - IPIP) e às escalas selecionadas do Inventário Fatorial de Personalidade (IFP). Todas as escalas de estilos interpessoais apresentaram correlações teoricamente esperadas com as demais medidas, contribuindo para a validade convergente dos construtos investigados. Os resultados encorajam o uso e a continuidade de estudos de validação do ESEI.

Palavras-chave: personalidade; psicometria; avaliação psicológica.

\section{ABSTRACT - Interpersonal styles scales: Evidence for convergent validity}

Interpersonal style is a multidimensional construct defined as the way an individual seeks for acceptance and social status. This research continues the validation studies of Inventory Interpersonal Styles Scales (ESEI) through the correlation of interpersonal style constructs with other personality measures. A sample of 848 individuals aged between 18 and 60 years old $(M=28.50), 328$ men and 520 women, living in southern and northeastern Brazil, answered, in person or through online platform (http://www.surveymonkey. com), the inventory ESEI, a measure of the five major personality factors (International Personality Item Pool - IPIP) and scales selected from the Factorial Personality Inventory (IFP). All interpersonal styles scales showed theoretically expected correlations with the other measures, contributing to the convergent validity of interpersonal style constructs. The results encourage the use and the continuity of validation studies of ESEI Inventory.

Keywords: personality; psychometrics; psychological assessment.

\section{RESUMEN - Escalas de Estilos Interpersonales: evidencias de validez convergente}

El estilo interpersonal es un constructo multidimensional definido como la forma en que un individuo busca aceptación y estatus social. Esa investigación prosiguió el estudio de validez del inventario 'Escalas de Estilos Interpersonales' (ESEI) a través de su correlación con otras medidas de personalidad. Participaron 848 personas con edades entre 18 y 60 años $(M=28,50), 328$ hombres y 520 mujeres, residentes en el Sur y Noreste de Brasil, que contestaron personalmente o en línea (www.surveymonkey.com) el Inventario ESEI, que es una medida de los cinco grandes factores de personalidad (International Personality Item Pool - IPIP), y las escalas seleccionadas del Inventario Factorial de Personalidad (IFP). Todas las escalas de estilos interpersonales mostraron correlaciones teóricamente esperadas con las otras medidas, lo que contribuye a la validez convergente de los constructos investigados. Los resultados estimulan el uso y la continuidad de los estudios de validez del ESEI.

Palabras clave: personalidad; psicometría; evaluación psicológica.

Os estilos interpessoais são as estratégias utilizadas pelos indivíduos para solucionar problemas no âmbito social. Acredita-se que, com base nas suas primeiras experiências e observações no contexto da infância, o indivíduo desenvolve estratégias próprias para inserirse no ambiente social e conquistar aceitação e status. Essa estratégia permanece na vida adulta como sua forma habitual de buscar inserção e influência nas comunidades de que participa (Roberts \& Wood, 2006).
A avaliação dos estilos interpessoais tem sido empregada em diversos campos teóricos e aplicados da psicologia. A influência dos estilos interpessoais de técnicos desportivos sobre o comportamento de atletas tem sido objeto de inúmeros estudos (Balaguer et al., 2012). Igualmente, destacam-se os estudos sobre a influência do estilo interpessoal de lideres, gestores e professores sobre o desempenho e o bem-estar de trabalhadores e estudantes, bem 
como sobre as intervenções para o desenvolvimento de estilos interpessoais mais efetivos nesses aspectos (Tessier, Sarrazin, \& Ntoumanis, 2010; Borghans, ter Weel, \& Weinber, 2008; Hunt \& Baruch, 2003). $\mathrm{O}$ enfoque interpessoal possui uma longa história nos estudos da personalidade e da psicopatologia (Pearson, Watkins, \& Mullan, 2010).

O Inventário Escalas de Estilos Interpessoais (ESEI) apresenta um conjunto de medidas psicométricas que revelou cobertura satisfatória das dimensões descritivas de estilo interpessoal apresentadas na literatura (Magalhães, 2013).

Esta pesquisa deu continuidade aos estudos de evidências de validade das escalas de estilos interpessoais, a fim de corroborar e refinar o entendimento do significado psicológico dessas escalas por meio da correlação com outras medidas psicológicas. Em primeiro lugar, será apresentado um breve histórico do enfoque interpessoal de avaliação das diferenças individuais. A seguir, serão esclarecidos o referencial teórico e as demais características do ESEI. Por fim, descrever-se-ão as medidas de personalidade a serem utilizadas para validação convergente.

\section{0 enfoque interpessoal na avaliação da personalidade}

Kiesler (1996) e Kiesler e Schmidt (2006) creditam a Sullivan (1953) a primeira sistematização de uma teoria interpessoal do comportamento. Para Sullivan (1953), o ser humano está primariamente motivado para obter segurança, manter sua autoestima e evitar sentimentos de ansiedade que possam resultar das relações interpessoais. O autor afirma que a ansiedade tem origem na insegurança associada à dependência interpessoal prolongada da criança em relação a seus cuidadores. As formas características de o indivíduo dominar sua ansiedade foram denominadas de "operações de segurança" (Sullivan, 1953).

As ideias de Sullivan (1953) foram incorporadas por Leary (2004) em sua teoria interpessoal da personalidade. Leary (2004) pensa que os aspectos mais importantes do comportamento humano são de natureza interpessoal e que, para compreender um indivíduo, é primordial obter informações sobre as estratégias que ele usa para lidar com as demais pessoas.

Nessa visão, a personalidade é um conjunto organizado de reflexos interpessoais recorrentes nas interações sociais de um indivíduo. Kiesler e Schmidt (2006) seguem essa perspectiva e sustentam que o comportamento humano somente pode ser compreendido em relação aos seus contextos interpessoais pregressos e atuais. Os autores afirmam que o comportamento busca evitar a ansiedade e realçar a autoestima e que medidas de personalidade são capazes de avaliar, no mesmo contínuo, polaridades disfuncionais e adaptativas do comportamento interpessoal. Além disso, consideram que a avaliação do estilo interpessoal requer ampla coleção de medidas específicas que sejam sistematicamente relacionadas entre si, a fim de que se possa identificar o padrão de comportamentos sociais que o indivíduo utiliza. Portanto, o estilo interpessoal é a maneira habitual de o indivíduo buscar inserção social, e esta deve ser avaliada mediante diversas dimensões descritivas (Kiesler, 1996; Kiesler \& Schmidt, 2006).

Nesse sentido, o ESEI pretende oferecer um conjunto de medidas psicológicas que, interpretadas de forma integrada, possam identificar os aspectos essenciais do estilo interpessoal do respondente.

\section{O Inventário Escalas de Estilos Interpessoais}

A construção do ESEI (Magalhães, 2013) partiu da premissa de que as experiências infantis têm impacto profundo na vida adulta e que cada indivíduo processa essas primeiras experiências para criar uma maneira consistente de lidar com as demandas da vida.

Essas ideias são comuns a diversas teorias do desenvolvimento da personalidade (Adler, 2005). Nesse sentido, o ESEI é composto de assertivas sobre relacionamentos e situações sociais vivenciados na infância, nos contextos da família, da escola e das amizades. O cabeçalho do instrumento estabelece o início de todas as sentenças com o texto "Quando eu era criança, eu...", que tem continuidade, em cada item, na descrição de comportamentos específicos. O respondente deve pontuar seu grau de concordância com as descrições de suas experiências interpessoais infantis. O uso da memória autobiográfica como técnica de avaliação da personalidade tem uma longa história, porém a operacionalização psicométrica dessa abordagem foi pouco desenvolvida.

Em linhas gerais, pesquisadores (Bruhn, 1995; Pillemer, 2003; Tulving, 2005) concordam que a memória humana segue os mesmos princípios seletivos e construtivos da percepção, sendo constituída por esquemas sobre o mundo. Nessa perspectiva, mais do que serem responsáveis pelo acúmulo de informações, os registros mnemônicos revelam atitudes e crenças derivadas das experiências passadas. Essas atitudes e crenças estão ativas no presente, e um processo de justificação regula a reconstrução dos eventos passados para que os "fatos" se apresentem consistentes com a atitude original do processo de recordação.

Acredita-se que a percepção do indivíduo acerca de seus comportamentos sociais na infância reflete crenças e atitudes ativas nas manifestações comportamentais presentes, conferindo-lhes a consistência característica de determinado estilo interpessoal. Portanto, a construção do ESEI insere-se em uma tradição de pesquisa sobre o uso da memória autobiográfica na avaliação da personalidade. Nessa mesma abordagem, encontra-se o inventário 
Basic Adlerian Scales for Interpersonal Success-Adult (BASIS-A) (Curlette, Wheeler, \& Kern, 1997). O ESEI (Magalhães, 2013) e o BASIS-A (Curlette et al., 1997) foram elaborados partindo da premissa de que o estilo interpessoal se baseia nas percepções da criança a respeito de suas interações sociais. A criança interpreta essas primeiras experiências da vida e desenvolve um sistema de crenças no que concerne às relações sociais e uma maneira consistente de agir que permanecem na vida adulta. Esse é o modus operandi individual, ou estilo interpessoal, para enfrentamento das demandas da convivência, com o objetivo de obter aceitação e status social.

Perguntas sobre lembranças infantis possuem vantagens importantes quanto a questões condizentes a comportamentos ou atitudes atuais do respondente. Ao se referir a eventos da infância, tais memórias reduzem a defensividade que pode emergir diante da possibilidade de relatar comportamentos considerados negativos ou inadequados na vida presente. As pessoas estão mais dispostas a admitir características negativas se estas são localizadas em um "passado distante” da infância (Curlette et al., 1997).

As categorias de estilo interpessoal descritas na literatura (Leary, 2004; Kiesler \& Schmidt, 2006; Wiggins, 1995) e as ideias seminais de Adler (2005) foram utilizadas como guias conceituais para a elaboração dos itens das escalas do ESEI. Na pesquisa de Magalhães (2013), o instrumento revelou uma estrutura fatorial consistente composta por nove fatores, com autovalores adequados (eigenvalue $\geq 1,70$ ) e
48,29\% da variância explicada. A Tabela 1 apresenta, com base nas conclusões do estudo de Magalhães (2013), a descrição das expectativas teóricas sobre o significado dos escores elevados em cada escala de estilo interpessoal.

\section{Evidências de validade convergente do Inventário Escalas de Estilos Interpessoais}

O objetivo desta pesquisa foi investigar evidências de validade de construto para as escalas de estilo interpessoal componentes do ESEI. Mediante verificação da adequação teórica das correlações entre as escalas de estilos interpessoais e outras medidas de personalidade, averiguou-se a validade convergente. As medidas de personalidade escolhidas foram os marcadores dos cinco grandes fatores de personalidade e escalas selecionadas do Inventário Fatorial de Personalidade (IFP). A taxonomia de traços mais aceita e utilizada propóe que a personalidade pode ser sumariada por meio de cinco dimensões amplas e ortogonais, rotuladas de neuroticismo, extroversão, abertura, sociabilidade e realização (Goldberg, 1992). Esse modelo é internacionalmente conhecido como Big Five (os cinco grandes fatores de personalidade, em denominação brasileira).

Sobre o significado geral desses fatores, neuroticismo refere-se à predisposição para experimentar afetos negativos, tais como ansiedade, raiva e depressão. Extroversão é tipicamente descrita como espírito gregário e assertividade. Abertura consiste no interesse por sentimentos e novas ideias, bem como na

Tabela 1

Significado dos escores elevados em cada escala do Inventário Escalas de Estilos Interpessoais

Escalas de

Estilos Interpessoais

Cautela

Intitulação

Pertencimento

Conformidade

Superioridade

Comando

Confrontação

Busca de reconhecimento Subescalas
Definição teórica do construto e exemplos de itens

Percepção desfavorável do ambiente social e vigilância interpessoal, por exemplo, "pensava que não gostavam de mim".

Expectativa de tratamento especial, de ter necessidades supridas por outrem, por exemplo, "atendiam a tudo que eu pedia".

Busca de contato interpessoal, sentimentos de confiança, inclusão e aceitação em relação aos outros, por exemplo, " possuía muitos amigos".

Uso de modelos externos para guiar o comportamento, por exemplo, "era disciplinado".

Desejo de demonstrar status superior para compensar sentimento de inferioridade, por exemplo, "preferia a companhia dos adultos".

Busca por controle das situações interpessoais e desejo de liderança, por exemplo, "queria comandar as brincadeiras".

Busca por independência de opinião e comportamento, desejo de impor as próprias vontades, por exemplo, "vingava-me quando me sentia prejudicado".

Busca de sucesso: necessidade de realização, de reconhecimento e de status, por exemplo, "queria me destacar pela minha inteligência";

Busca de aprovação: necessidade de sentir-se querido e aceito, por exemplo, "esforçava-me para agradar os adultos". 
demonstração de sensibilidade estética e flexibilidade. A sociabilidade engloba aspectos de altruísmo, cuidado, confiança e cooperação. Por fim, realização inclui persistência, responsabilidade e necessidade de realização (McCrae \& Costa, 2003).

O projeto internacional de pesquisa em personalidade, denominado International Personality Item Pool - IPIP (http://ipip.ori.org/), liderado por Goldberg et al. (2006), oferece livre acesso a medidas de diferenças individuais e convida pesquisadores a utilizarem os materiais compartilhados e disseminarem seus achados, de modo a avançar no desenvolvimento da área de avaliação da personalidade. O site disponibiliza dois questionários, um com 50 e outro com 100 itens, para avaliação dos cinco grandes fatores de personalidade. A presente pesquisa utilizou a versão de 50 itens, traduzida e adaptada para o contexto brasileiro por Brockveld (2013), com 10 itens para cada fator.

O IFP foi elaborado por Pasquali, Azevedo e Ghesti (1997), com base no Edwards Personal Preference Schedule (EPPS), desenvolvido por Allen L. Edwards em 1953 e revisto em 1959, fundamentado na teoria das necessidades básicas de Murray (1938). A adaptação brasileira não se restringiu à tradução do original, mas incluiu reformulações visando responder a críticas apresentadas anteriormente ao EPPS, justificando a mudança do nome original do teste. O IFP foi aplicado a 3.399 sujeitos $(33 \%$ homens e $67 \%$ mulheres), a maioria composta por estudantes universitários (49,5\%) oriundos de 11 estados brasileiros. O instrumento avalia o indivíduo normal em 15 necessidades ou motivos psicológicos. Para a presente pesquisa, foram selecionados 10 fatores do IFP que demonstraram melhores evidências de validade e precisão, conforme informações apresentadas no manual do instrumento (Pasquali et al., 1997). A Tabela 2 exibe os fatores selecionados e as respectivas descrições do significado dos altos escores.

Espera-se que as correlações entre o ESEI, as escalas do IPIP e as escalas selecionadas do IFP revelem associações coerentes com as expectativas teóricas sobre o significado das escalas de estilos interpessoais (Tabela 1 ).

\section{Método}

\section{Participantes}

Participaram da investigação 848 pessoas com idades entre 18 e 60 anos $(M=28,50 ; D P=5,35)$ 328 homens e 520 mulheres —, residentes nas Regiões Sul e Nordeste. A escolaridade predominante foi ensino superior incompleto $(63,33 \%)$, seguida por graduação superior completa $(21,66 \%)$ e pós-graduação $(15,01 \%)$.

\section{Instrumentos}

Os participantes responderam ao ESEI composto por 83 itens, distribuídos em oito escalas: pertencimento, cautela, superioridade, comando, intitulação, confrontação, conformidade e reconhecimento. A escala reconhecimento possui duas facetas: sucesso e aprovação.

Tabela 2

Fatores do Inventário Fatorial de Personalidade e descrição das características atribuídas a escores altos em cada escala

\begin{tabular}{|c|c|}
\hline Fatores do IFP & Significado dos escores altos \\
\hline Denegação & $\begin{array}{l}\text { Tendência a se entregar passivamente à força externa, aceitar castigo e culpa e resignar-se } \\
\text { ao destino, admitindo inferioridade. }\end{array}$ \\
\hline Intracepção & $\begin{array}{l}\text { Tendência a se deixar conduzir por sentimentos e julgamentos subjetivos; observação e } \\
\text { sensibilidade em relação ao comportamento das outras pessoas. }\end{array}$ \\
\hline Desempenho & $\begin{array}{l}\text { Necessidade de vencer obstáculos, de realizar ações difíceis e de executar tarefas } \\
\text { independentemente e com o máximo de rapidez. }\end{array}$ \\
\hline Exibição & Desejo de impressionar, de ser ouvido e visto, de entreter e fascinar as pessoas. \\
\hline Afago & Busca de apoio, proteção, amor, orientação, consolo e perdão. \\
\hline Agressão & $\begin{array}{l}\text { Necessidade de atacar, lutar, opor-se a algo ou alguém, mediante o uso da força, e revidar a } \\
\text { injúria. Desejo de superar com vigor e violência a oposição. }\end{array}$ \\
\hline Deferência & $\begin{array}{l}\text { Necessidade de admirar, prestigiar, apoiar, honrar, elogiar, imitar ou sujeitar-se a um } \\
\text { modelo ou superior, ou ainda conformar-se com os costumes e as tradições. }\end{array}$ \\
\hline Ordem & $\begin{array}{l}\text { Tendência a manter a ordem e a valorizar a limpeza, o equilíbrio e a precisão dos objetos } \\
\text { do mundo exterior. }\end{array}$ \\
\hline Desempenho & $\begin{array}{l}\text { Necessidade de vencer obstáculos, de realizar ações difíceis e de executar tarefas } \\
\text { independentemente e com o máximo de rapidez. }\end{array}$ \\
\hline Dominância & $\begin{array}{l}\text { Desejo de controlar o ambiente e o comportamento alheio por meio de sugestão, sedução, } \\
\text { persuasão e comando. }\end{array}$ \\
\hline
\end{tabular}

Nota: IFP=Inventário Fatorial de Personalidade. 
Para mesurar os cinco grandes fatores de personalidade, foi utilizado o IPIP desenvolvido por Goldberg et al. (2006), em sua versão de 50 itens traduzida e adaptada para o contexto brasileiro por Brockveld (2013). $\mathrm{Na}$ amostra investigada, o IPIP mostrou estrutura fatorial correspondente ao modelo dos cinco grandes fatores, com alfas de Cronbach entre 0,76 e 0,84. Foram selecionadas 10 escalas do IFP (denegação, intracepção, desempenho, exibição, afago, agressão, deferência, ordem, desempenho e dominância) (Pasquali et al., 1997). $\mathrm{Na}$ amostra averiguada, as escalas do IFP apresentaram alfas de Cronbach entre 0,69 e 0,79. Todos os instrumentos adotaram escala do tipo Likert de 5 pontos $(1=$ discordo totalmente; 5 = concordo totalmente).

\section{Procedimento}

A coleta de dados foi presencial em universidades, mediante autorização das autoridades acadêmicas competentes, ou por meio da plataforma on-line (http://www. surveymonkey.com).

A pesquisa foi aprovada pelo Comitê de Ética em Pesquisa da instituição de afiliação do pesquisador, sendo atendidas as determinações éticas da Resolução n. ${ }^{\circ}$ 196/1996 do Conselho Nacional de Saúde.

\section{Análise de dados}

Os dados coletados atenderam aos requisitos para uso de estatística paramétrica, permitindo o uso de correlações de Pearson para examinar as relações entre as escalas de estilos interpessoais e as demais medidas de personalidade.

\section{Resultados e Discussão}

O ESEI é composto por um conjunto de escalas correspondentes às variáveis de estilo interpessoal: cautela, intitulação, pertencimento, comando, superioridade, conformidade e busca de reconhecimento (subescalas busca de sucesso e busca de aprovação).

Esta pesquisa teve o objetivo de testar as expectativas teóricas relativas ao significado psicológico de cada escala de estilo interpessoal por intermédio do exame das correlações entre as escalas de estilo interpessoal e outras medidas de personalidade.

A Tabela 3 apresenta as correlações de Pearson entre as escalas de estilos interpessoais e os fatores selecionados do IFP.

A Tabela 4 expõe as correlações de Pearson entre as escalas de estilos interpessoais. São evidenciadas,

Tabela 3

Correlações de Pearson entre o Inventário Escalas de Estilos Interpessoais e os fatores do Inventário Fatorial de Personalidade

\begin{tabular}{lcccccccccc}
\hline & CAU & INT & PER & COM & CFR & SUP & CFM & BRE & BSU & BAP \\
\hline Agressividade & $0,36^{* *}$ & $0,20^{* *}$ & $-0,24^{* *}$ & $0,26^{* *}$ & $0,46^{* *}$ & $0,18^{*}$ & $-0,22^{* *}$ & - & - & - \\
Desempenho & - & - & - & $0,20^{* *}$ & - & - & - & $0,25^{* *}$ & $0,34^{*}$ & $0,20^{* *}$ \\
Ordem & - & - & $0,20^{*}$ & - & - & - & $0,33^{* *}$ & $0,26^{* *}$ & $0,25^{* *}$ & $0,17^{*}$ \\
Exibição & - & $0,28^{* *}$ & - & $0,61^{* *}$ & $0,29^{* *}$ & $0,26^{* *}$ & - & $0,50^{* *}$ & $0,40^{* *}$ & $0,53^{* *}$ \\
Assistência & - & - & - & $0,20^{* *}$ & - & - & $0,28^{* *}$ & $0,20^{*}$ & - & - \\
Afago & - & $0,45^{* *}$ & - & $0,20^{* *}$ & - & - & $0,29^{* *}$ & $0,32^{* *}$ & $0,19^{*}$ & $0,33^{* *}$ \\
Dominância & - & $0,40^{* *}$ & - & $0,58^{*}$ & $0,23^{*}$ & $0,20^{*}$ & - & $0,46^{* *}$ & $0,38^{* *}$ & $0,40^{* *}$ \\
Intracepção & - & - & - & - & - & - & $0,30^{* *}$ & $0,51^{* *}$ & $0,50^{* *}$ & $0,46^{* *}$ \\
Denegação & $0,42^{* *}$ & $0,34^{* *}$ & $-0,27^{* *}$ & - & - & $0,38^{* *}$ & - & $0,21^{*}$ & - & $0,36^{* *}$ \\
Deferência & - & - & $0,37^{* *}$ & - & - & $-0,25^{* *}$ & $0,47^{* *}$ & $0,46^{* *}$ & $0,45^{* *}$ & $0,35^{* *}$ \\
\hline
\end{tabular}

Nota: ${ }^{*} p<0,05 ;{ }^{* *} p<0,01$; CAU=cautela; INT=intitulação; PER=pertencimento; COM=comando; CFR=confrontação; SUP=superioridade; $\mathrm{CFM=conformidade;} \mathrm{BRE=busca} \mathrm{de} \mathrm{reconhecimento;} B S U=$ busca de sucesso; BAP=busca de aprovação.

Tabela 4

Correlações de Pearson entre o Inventário Escalas de Estilos Interpessoais e os cinco grandes fatores de personalidade

\begin{tabular}{lcccccccccc}
\hline & CAU & INT & PER & COM & CFR & SUP & CFM & BRE & BSU & BAP \\
\hline Extroversão & $-0,20^{* *}$ & - & $0,43^{* *}$ & $0,32^{* *}$ & - & - & - & $0,17^{*}$ & $0,23^{* *}$ & - \\
Realização & $-0,26^{* *}$ & - & $0,19^{* *}$ & - & - & - & $0,39^{* *}$ & $0,21^{* *}$ & $0,28^{* *}$ & - \\
Neuroticismo & $0,30^{* *}$ & - & $-0,42^{* *}$ & - & - & $0,17^{*}$ & - & - & - & - \\
Abertura & $-0,22^{* *}$ & - & $0,18^{* *}$ & $0,15^{*}$ & - & - & - & - & $0,29^{* *}$ & - \\
Sociabilidade & - & - & $0,30^{* *}$ & - & $-0,16^{*}$ & - & $0,20^{*}$ & $0,20^{*}$ & - & $0,24^{* *}$ \\
\hline
\end{tabular}

Nota: ${ }^{*} p<0,05 ;{ }^{* *} p<0,01 ;$ CAU=cautela; INT=intitulação; PER=pertencimento; COM=comando; CFR=confrontação; SUP=superioridade; $\mathrm{CFM=conformidade;} \mathrm{BRE=busca} \mathrm{de} \mathrm{reconhecimento;} \mathrm{BSU}=$ busca de sucesso; BAP=busca de aprovação. 
ainda, as medidas dos cinco grandes fatores de personalidade.

A escala cautela foi desenvolvida para mensurar o quanto o indivíduo declara uma experiência desfavorável no ambiente familiar e social da infância, revelando sua inclinação a comportamentos de vigilância interpessoal, desconfiança, ansiedade, agressividade e isolamento (Magalhães, 2013). No presente estudo, cautela correlacionou-se com neuroticismo $(r=0,30 ; p<0,01)$ e denegação $(r=0,42$; $p<0,01)$, sugerindo que indivíduos com altos escores em cautela tendem a ser preocupados, nervosos, inseguros e propensos ao sofrimento emocional. A correlação entre cautela e agressividade $(r=0,36$; $p<0,01)$ corrobora a descrição dessa dimensão de estilo interpessoal como uma tendência a perceber o ambiente social de maneira desfavorável, associada à vigilância e ao distanciamento interpessoal. Nesse sentido, Carmody, Crossen e Wens (1989) observaram correlações entre medidas de neuroticismo, agressividade e distanciamento interpessoal.

Ademais, cautela foi correlacionada negativamente com extroversão $(r=-0,20 ; p<0,01)$, realização $(r=-0,26 ; p<0,01)$ e abertura $(r=-0,22 ; p<0,01)$. Esse conjunto de correlações converge para a tendência a reduzir interações sociais e comportamentos exploratórios e sugere menor persistência e organização em relação a responsabilidades e compromissos. Gallo, Smith e Ruiz (2003) verificaram esse mesmo padrão de correlações entre os cinco grandes fatores de personalidade e estilos interpessoais propensos à ansiedade e ao isolamento social.

Da mesma forma, na pesquisa de Shaver e Brennan (1992) houve relação inversa entre os estilos apego ansioso e evitativo e os traços de extroversão, sociabilidade e realização; e relação positiva com neuroticismo. McCrae e Costa (1989) relatam que os traços extroversão e abertura possuem relação inversa com um estilo interpessoal distante-introvertido ou frio, tal como observado no presente estudo no tocante à escala cautela. Portanto, as correlações encontradas entre a escala cautela e o modelo dos cinco grandes fatores correspondem a inclinações para a insegurança e o isolamento social, confirmando expectativas teóricas relacionadas a essa medida.

Quanto à escala comando, espera-se que esteja associada ao desejo de liderar e/ou controlar as decisões e ações grupais (Magalhães, 2013). No presente estudo, as correlações com exibição $(r=0,61$; $p<0,01)$, dominância $(r=0,58 ; p<0,01)$ e agressividade $(r=0,26 ; p<0,01)$ atendem a essas expectativas teóricas. Houve também correlações com desempenho, assistência e afago $(r=0,20 ; p<0,01)$, dimensões que, embora de baixa magnitude, matizam o construto comando com aspectos de produtividade, desejo de ajudar e de ser gostado. Ademais, a correlação com extroversão $(r=0,32 ; p<0,01)$ agrega inclinações para comportamento gregário e assertividade, aspectos que favorecem a assunção de papéis de liderança nas relações sociais.

Na pesquisa de Forrester e Tashchian (2013), indivíduos com escores elevados em extroversão mostraram inclinações para uma abordagem dominadora de resolução de conflitos, por meio do uso de influência, autoridade e poder para ter as decisões finais tomadas a seu favor. Anteriormente, McCrae e Costa (1989) observaram a correlação entre um estilo interpessoal seguro-dominante e extroversão. Por conseguinte, aqui a correlação encontrada entre a medida de extroversão e a dimensão de estilo interpessoal comando corrobora esses achados e apoia o significado psicológico teoricamente atribuído a essa dimensão de estilo interpessoal por Magalhães (2013).

O fator dominância do IFP significa o desejo de controlar o ambiente e o comportamento alheio por intermédio da sugestão, da persuasão e do comando (Pasquali et al., 1997). Houve correlação entre comando (ESEI) e dominância (IFP) no presente estudo $(r=0,58 ; p<0,01)$, sugerindo que pessoas com escores elevados em comando desejam controlar o ambiente e outras pessoas. Nesse sentido, Curlette et al. (1997) informam correlações entre a dimensão assumir o comando (taking charge) do inventário BASIS-A e uma medida de dominância nas relações sociais $(r=0,59$; $p<0,01)$. Primi, Moggi e Casellato (2004) investigaram relações entre o IFP e o Inventário de Busca Autodirigida (Mansão, 2005), que mensura interesses ocupacionais de acordo com o modelo de Holland (1997). O interesse empreendedor, característico de indivíduos com inclinações a usar a persuasão e o comando para controlar outras pessoas, correlacionou-se com os fatores dominância $(r=0,39)$, exibição $(r=0,25)$ - desejo de impressionar, ser ouvido e visto, de fascinar as pessoas - e agressão $(r=0,29)$, ou seja, desejo de superar com vigor e violência a oposição, do IFP. Na pesquisa de Magalhães (2006), o interesse empreendedor, medido pelo Inventário de Busca Autodirigida, foi associado a escores mais elevados na escala assumir o comando do BASIS-A. Logo, as correlações encontradas no presente estudo entre a escala comando (ESEI) e o IFP mostram-se consistentes com achados de outros estudos que investigaram essas variáveis de personalidade.

A escala de busca de reconhecimento foi desenvolvida para avaliar o desejo de atender às expectativas externas de figuras de autoridade e/ou aquisição de status por meio do comportamento adequado e/ou de desempenho diferenciado na realização de tarefas relacionadas às expectativas sociais (Magalhães, 2013). Assim, estima-se que quanto mais elevados forem os escores nessa escala, maior será a preocupação em apresentar comportamentos que resultem na aprovação externa e/ 
ou na conquista de atenção social pelo atendimento ou superação de expectativas de desempenho. As correlações obtidas entre busca de reconhecimento e as escalas desempenho $(r=0,25 ; p<0,01)$ e intracepção $(r=0,51$; $p<0,01)$ do IFP confirmam essa expectativa. O fator desempenho (IFP) mensura o desejo de realizar tarefas de modo independente e com rapidez, buscando dominar objetos, pessoas e ideias; e o fator intracepção indica o quanto o indivíduo se dedica à observação e análise dos comportamentos e sentimentos dos outros (Pasquali et al., 1997).

Pesquisas revelam que a capacidade de decodificar o comportamento não verbal de outras pessoas é mais desenvolvida em indivíduos com estilos interpessoais dependentes e voltados para agradar outras pessoas (Kato, 2012). Conclui-se que, quanto mais o indivíduo deseja agradar outras pessoas e obter reconhecimento, mais será atento a pistas não verbais que indiquem os estados subjetivos alheios e informem o impacto do seu comportamento no ambiente social. Nesse sentido, a correlação entre busca de reconhecimento e intracepção $(r=0,51 ; p<0,01)$ corrobora a expectativa teórica relacionada à referida dimensão de estilo interpessoal.

Em Magalhães (2013), análises fatoriais revelaram que a escala busca de reconhecimento é composta de duas facetas: busca de sucesso e busca de aprovação. A faceta busca de sucesso refere-se a contextos que demandam desempenho e cumprimento de tarefas, como, por exemplo, os ambientes de trabalho e acadêmico, estando também ligada à ambição e ao desejo de status (Magalhães, 2013). Essa faceta apresentou correlações com os fatores desempenho $(r=0,34 ; p<0,01)$ e intracepção $(r=0,50 ; p<0,01)$ do IFP. Portanto, os escores elevados em busca de sucesso parecem apontar o desejo de apresentar desempenho diferenciado e atento às expectativas externas. Ademais, as correlações com os fatores extroversão $(r=0,28 ; p<0,01)$, realização $(r=0,23 ; p<0,01)$ e abertura $(r=0,29 ; p<0,01)$ indicam aspectos de assertividade, disciplina e curiosidade. Esses dados apoiam o significado psicológico atribuído à faceta busca de sucesso de acordo com o modelo teórico do enfoque interpessoal da personalidade.

A faceta busca de aprovação expressa a necessidade de aceitação pelos outros, de ser aprovado como pessoa digna de amor (Magalhães, 2013). As correlações com afago $(r=0,33 ; p<0,01)$ (IFP) e com sociabilidade $(r=0,24 ; p<0,01)$, ausentes na dimensão busca de sucesso, corroboram a diferença sugerida entre as duas facetas. O fator afago significa busca de apoio, proteção, amor, orientação, consolo e perdão (Pasquali et al., 1997), sendo a correlação encontrada consistente com a expectativa teórica. Forrester e Tashchian (2013) observam que o traço sociabilidade está associado a um estilo interpessoal cooperativo e avesso a conflitos. McCrae e Costa (1989) notam associação entre o estilo interpessoal denominado de "pouco seguro-submisso" e o traço sociabilidade. Esses dados confirmam a correlação aqui encontrada entre sociabilidade e busca de aprovação. A faceta busca de aprovação também apresentou correlações com os fatores desempenho $(r=0,20 ; p<0,01)$ e intracepção $(r=0,46 ; p<0,01)$ do IFP, sendo essas associações interpretadas da mesma forma que as achadas entre desempenho, intracepção e busca de reconhecimento.

A escala intitulação foi desenvolvida para revelar o uso ou a simulação de vulnerabilidade pessoal para despertar sentimentos de compaixão em outras pessoas com o objetivo de obter privilégios e colocar os demais a seu serviço, como, por exemplo, "usava minha fragilidade para obter o que desejava dos outros" (Magalhães, 2013). Essa escala mostrou correlações com afago $(r=0,45 ; p<0,01)$, dominância $(r=0,40$; $p<0,01)$ e denegação $(r=0,34 ; p<0,01)$, comprovando que a intitulação articula a busca do indivíduo por apoio emocional e cuidados (afago) com o desejo de controlar outras pessoas, de tal modo que estas venham a prover as suas necessidades (dominância). Ainda, agrega-se a esse cenário a baixa autoconfiança, inerente ao sentimento de incapacidade de dar conta das próprias necessidades (denegação).

No ESEI, a escala superioridade revela um conceito fundamental das ideias de Adler (2005): a busca compensatória de status superior associada a sentimentos de menos-valia. Espera-se que escores elevados nesse fator estejam ligados à baixa autoestima e à ansiedade social, sentimentos que o indivíduo tenta combater por meio de um estilo interpessoal caracterizado pelo controle da autoapresentação e pela expressão de atitudes e comportamentos que ele considera associados à condição de superioridade social (Magalhães, 2013). Essa conceituação foi apoiada pelas seguintes correlações com o IFP: denegação $(r=0,38 ; p<0,01)$, no tocante à baixa autoestima $\mathrm{e}$ aos sentimentos de inferioridade; exibição $(r=0,26$; $p<0,01)$ e dominância $(r=0,20 ; p<0,05)$, quanto ao desejo de impressionar e controlar os demais; agressividade $(r=0,18 ; p<0,05)$, indicando o desejo de se impor pela força; e deferência $(r=-0,25 ; p<0,01)$, revelando aversão por posições subalternas e comportamentos de reverência a autoridades. Por fim, apresentou correlação positiva com o fator neuroticismo da Bateria Fatorial de Personalidade (BFP) $(r=0,17 ; p<0,05)$, corroborando que o desejo de superioridade tende a associar-se a emoções negativas.

Em relação à escala pertencimento, acredita-se que signifique o uso de estratégias interpessoais cooperativas e pacíficas concernentes aos sentimentos de confiança, inclusão e aceitação em relação aos demais (Magalhães, 2013). As correlações positivas com extroversão 
$(r=0,43 ; p<0,01)$ e sociabilidade $(r=0,30 ; p<0,01)$ e negativa com neuroticismo $(r=-0,42 ; p<0,01)$ apoiam essa interpretação dos escores de pertencimento.

A escala pertencimento/interesse social do inventário BASIS-A possui a mesma definição constitutiva da escala pertencimento do ESEI e correlacionou positivamente com extroversão (Myers-Briggs Type Indicator - MBTI) e negativamente com depressão (Beck Depression Inventory - BDI) (Kern, Gormley, \& Curlette, 2008). As correlações negativas com denegação $(r=-0,27 ; p<0,01)$ e agressividade $(r=-0,24$; $p<0,01)$ corroboram a hipótese de que indivíduos com escores elevados em pertencimento sejam menos propensos tanto ao uso da força para impor seus desejos quanto à submissão a forças externas, buscando construir relacionamentos de cooperação e equivalência entre pares.

Sobre a escala confrontação, os escores elevados teoricamente estão associados à agressividade e à competitividade, bem como à busca por independência de opinião e comportamento. Essa interpretação foi apoiada pelos resultados, destacando-se a correlação com agressividade $(r=0,46 ; p<0,01)$, além da associação positiva com exibição $(r=0,29 ; p<0,01)$ e dominância $(r=0,23 ; p<0,01)$ e da relação inversa com sociabilidade $(r=-0,16 ; p<0,05)$. Esta última correlação confirma a relação inversa entre sociabilidade e um estilo dominante de resolução de conflitos observada por Forrester e Tashchian (2013) e por McCrae e Costa (1989).

No ESEI, a escala conformidade é formada por itens que descrevem inclinações à disciplina e à obediência (Magalhães, 2013). Está, portanto, teoricamente associada à propensão para apresentar comportamentos socialmente desejáveis. Com base no modelo teórico que fundamentou o ESEI, esperase que o indivíduo com altos escores nesse fator tenda a respeitar as autoridades e as convenções sociais.

No presente estudo, essas expectativas foram corroboradas nas correlações com as escalas deferência $(r=0,47 ; p<0,01)$ e ordem $(r=0,33 ; p<0,01)$ do IFP e com a escala consciência $(r=0,39 ; p<0,01)$. O fator deferência (IFP) está relacionado à conformidade aos costumes e à reverência a figuras de autoridade e prestígio social. A deferência expressa o desejo de dar suporte, admirar, elogiar e honrar os superiores, bem como imitá-los e obedecê-los. O fator consciência, por sua vez, refere-se a comportamentos de responsabilidade, organização, planejamento e disciplina, uma conceituação similar ao significado psicológico dos escores de ordem (IFP). Desse modo, o entendimento da dimensão conformidade serve como medida indicadora de responsabilidade e disciplina nas relações interpessoais.

Outras correlações significativas foram encontradas entre conformidade e os fatores do IFP: intracepção $\quad(r=0,30 ; \quad p<0,01), \quad$ afago $\quad(r=0,29$; $p<0,01)$, assistência $(r=0,28 ; p<0,01)$, agressividade $(r=-0,22 ; p<0,01)$ e sociabilidade $(r=0,20 ; p<0,01)$. Essas correlações, embora de menor magnitude, descrevem comportamentos que convergem para a definição constitutiva de conformidade em termos do apreço por aceitação social e em posturas de generosidade e compaixão (assistência e sociabilidade). A correlação negativa com agressividade apoia essa descrição, bem como a necessidade de afago e a preferência pela intracepção. A relação com intracepção sugere que a conformidade esteja ligada à observação das demandas sociais e dos aspectos do ambiente externo. Nos itens da escala de conformidade, indivíduos com altos escores afirmam que, quando crianças, eram disciplinados e ofereciam bom exemplo. Nesse sentido, tal lembrança aponta também a busca por apoio e proteção dos adultos, o que se revela na correlação com afago (IFP).

A construção do ESEI partiu da premissa de que as interações sociais na infância formam inclinações comportamentais que perduram no indivíduo adulto em sua busca por aceitação e influência nas relações sociais. Os resultados corroboram essa ideia e a utilidade da memória autobiográfica na avaliação da personalidade. Todas as escalas de estilos interpessoais apresentaram correlações teoricamente esperadas com as medidas utilizadas para validação convergente, confirmando e aperfeiçoando o entendimento dos construtos de estilo interpessoal. Os resultados encorajam o uso e a continuidade de estudos de validação das escalas de estilos interpessoais. Ademais, recomenda-se explorar o potencial de aplicação do ESEI em intervenções psicológicas.

No âmbito da psicologia clínica, acredita-se que o ESEI possa ser útil para avaliar a compatibilidade entre paciente e psicoterapeuta, bem como auxiliar na adequação de estratégias psicoterapêuticas a pacientes com diferentes estilos interpessoais. Por exemplo, espera-se que a escala confrontação seja um critério importante para recomendar maior ou menor diretividade das intervenções psicoterapêuticas. Pensa-se que pacientes com pontuações elevadas nessa escala, associadas à preocupação com independência e controle, prefiram uma abordagem predominantemente não diretiva, ao menos até que uma aliança terapêutica seja consolidada.

A eficácia do uso do ESEI na facilitação do processo psicoterapêutico propriamente dito mostra-se promissora. $\mathrm{O}$ uso da memória autobiográfica na avaliação da personalidade facilita, no indivíduo avaliado, a construção de conexões entre experiências da infância e comportamentos na vida adulta. Desse modo, espera-se que o ESEI venha a favorecer o insight do paciente sobre suas dificuldades socioemocionais e facilitar mudanças. Portanto, recomenda-se 
o teste de hipóteses relacionadas ao uso do ESEI na prática psicoterápica.

No âmbito da psicologia organizacional, o ESEI pode ser utilizado em investigações acerca do desempenho no trabalho. Por exemplo, é provável que aspectos de complementaridade entre os estilos interpessoais influenciem a produtividade de equipes de trabalho (Cowley, Nauer, \& Anderson, 2015).

O psicólogo brasileiro carece de instrumentos para investigação de diferenças individuais com aplicação direta em processos de intervenção psicológica. O ESEI foi desenvolvido para oferecer uma avaliação do componente interpessoal da personalidade a fim de instrumentar o trabalho do psicólogo nos diversos contextos de sua atuação, em que necessite prover não somente entendimento, mas principalmente auxiliar na aquisição de comportamentos mais eficazes diante das demandas do trabalho, da convivência social e das relações de intimidade.

O presente estudo encoraja novas pesquisas com o ESEI, de modo a consolidar as interpretações construídas até então a respeito do significado das pontuações obtidas nas escalas que compõem o inventário e a avançar para a definição das possibilidades de uso desse instrumento na prática profissional do psicólogo.

\section{Referências}

Adler, A. (2005). The collected clinical works of Alfred Adler: The neurotic character. Washington: Alfred Adler Institute. v. 1.

Balaguer, I., González, L., Fabra, P., Castillo, I., Mercé, J., \& Duda, J. L. (2012). Coaches' interpersonal style, basic psychological needs and the well- and ill-being of young soccer players: A longitudinal analysis. Journal of Sports Sciences, 30(15), 1619-1629. doi: 10.1080/02640414.2012.731517

Borghans, L., ter Weel, B., \& Weinberg, B. A. (2008). Interpersonal styles and labor market outcomes. Journal of Human Resources, 43(4), 815858. doi: $10.3386 / \mathrm{w} 12846$

Brockveld, K. (2013). The relationship between five factor model personality traits and social anxiety: A comparison between Australia and Brazil. (Tese de doutorado não publicada), Macquarie University, Sidney, Australia.

Bruhn, A. R. (1995). Early memories in personality assessment. Em J. N. Butcher (Ed.), Clinical personality assessment: Practical approaches. (pp. 278-301). Nova York: Oxford University Press.

Carmody, T. P., Crossen, J. R., \& Wens, A. N. (1989). Hostility as a health risk factor: Relationships with neuroticism, type A behavior, attentional focus, and interpersonal style. Journal of Clinical Psychology, 45(5), 754-762.doi: 10.1002/1097-4679(198909)45:5<754::AIDJCLP2270450510>3.0.CO;2-C

Curlette, W., Wheeler, M., \& Kern, R. (1997). BASIS-A Inventory: Technical manual. Highlands, NC: TRT.

Cowley, J. A., Nauer, K. S., \& Anderson, B. R. (2015). Emergent relationships between team member interpersonal styles and cybersecurity team performance. Procedia Manufacturing, 3, 5110-5117. doi: 10.1016/j.promfg.2015.07.526

Forrester, W. R., \& Tashchian, A. (2013). Effects of personality on conflict resolution in student teams: A structural equation modeling approach. Journal of College Teaching \& Learning, 10(1), 39-46.

Gallo, L. C., Smith, T. W., \& Ruiz, J. M. (2003). An interpersonal analysis of adult attachment style: Circumplex descriptions, recoiled developmental experiences, self-representations, and interpersonal functioning in adulthood. Journal of Personality, 71(2), $141-181$.

Goldberg, L. R. (1992). The development of markers for the Big-Five factor structure. Psychological Assessment, 4(1), $26-42$.

Goldberg, L. R., Johnson, J. A., Eber, H. W., Hogan, R., Ashton, M. C., Cloninger, C. R., \& Gough, H. C. (2006). The International Personality Item Pool and the future of public-domain personality measures. Journal of Research in Personality, 40, 84-96. doi: 10.1016/j. jrp.2005.08.007

Holland, J. L. (1997). Making vocational choices: A theory of vocational personalities and work environments. Odessa, FL.: PAR.

Hunt, J. W., \& Baruch, Y. (2003). Developing top managers: The impact of interpersonal skills training. Journal of Management Development, 22(8), 729-752. doi: 10.1108/02621710310487882

Kato, M. (2012). The effects of individual differences and self-consciousness on nonverbal decoding accuracy. Wellesley College - Honors Thesis Collection, Paper 27. Recuperado de http://repository.wellesley.edu/thesiscollection.

Kern, R. M., Gormley, L., \& Curlette, W. L. (2008). BASIS-A Inventory empirical studies: research findings from 2000 to 2006. Journal of Individual Psychology, 64(3), 280-309.

Kiesler, D. J. (1996). Contemporary interpersonal theory and research: Personality, psychopathology and psychotherapy. Nova York: Wiley.

Kiesler, D. J., \& Schmidt, J. A. (2006). The impact message inventory - circumplex (IMI-C) manual: Manual, instrument, scoring guide, and worksheets. Redwood City: Mind Garden.

Leary, T. (2004). Interpersonal diagnosis of personality: A functional theory and methodology for personality evaluation. Eugene: Resource Publications. Magalhães, M. O. (2006). Relação entre personalidades vocacionais e estilos interpessoais. Revista Brasileira de Orientação Profissional, 7, 11-22.

Magalhães, M. O. (2013). Escalas de Estilos Interpessoais (ESEI): construção, validade fatorial e consistência interna. Psicologia: Reflexão e Crítica, 26(4), 627-635. doi: 10.1590/S0102-79722013000400002

Mansão, C. S. M. (2005). Interesses profissionais: validação do Self-Directed Search Career Explorer-SDS. (Tese de doutorado não publicada). Pontifícia Universidade Católica de Campinas, Campinas, SP, Brasil.

McCrae, R. R., \& Costa, P. T. (1989). The structure of interpersonal traits: Wiggins's circumflex and the Five-Factor Model. Journal of Personality and Social Psychology, 56, 586-595.

McCrae, R. R., \& Costa, P. T. (2003). Personality in adulthood: A five-factor theory perspective. Nova York: Guilford Press. 
Murray, H. A. (1938). Explorations in personality: A clinical and experimental study of fifty men of college age. New York: Oxford University Press.

Pasquali, L., Azevedo, M. M., \& Ghesti, I. (1997). Inventário Fatorial de Personalidade: Manual técnico e de avaliação. São Paulo: Casa do Psicólogo.

Pearson, K. A., Watkins, E. R., \& Mullan, E. G. (2010). Submissive interpersonal style mediates the effect of brooding on future depressive symptoms. Behaviour Research and Therapy, 48(10), 966-973. doi: 10.1016/j.brat.2010.05.029

Pillemer, D. B. (2003). Directive functions of autobiographical memory: The guiding power of the specific episode. Memory, 11(2), 193-202. DOI: $10.1080 / 741938208$

Primi, R., Moggi, M. A., \& Casellato, E. O. (2004). Estudo correlacional do Inventário de Busca Auto Dirigida (Self-Directed Search) com o IFP. Psicologia Escolar Educacional, 8(1), 47-54. doi: 10.1590/S1413-85572004000100006

Roberts, B. W., \& Wood, D. (2006). Personality development in the context of the neo-socioanalytic model of personality. Em: D. K. Mroczek \& T. D. Little (Orgs.), Handbook of personality development. (pp. 11-39). Mahwah, NJ: Erlbaum.

Shaver, P. R., \& Brennan, K. A. (1992). Attachment styles and the "big five" personality traits: Their connections with each other and with romantic relationship outcomes. Personality and Social Psychology Bulletin, 18(5), 536-545.

Sullivan, H. S. (1953). The interpersonal theory of psychiatry. Nova York: Norton.

Tessier, D., Sarrazin, P., \& Ntoumanis, N. (2010). The effect of an intervention to improve newly qualified teachers' interpersonal style, students motivation and psychological need satisfaction. Contemporary Educational Psychology, 35(4), 242-253.

Tulving, E. (2005). Episodic memory and autonoesis: Uniquely human? Em: H. S. Terrace \& J. Metcalfe (Eds.), The missing link in cognition: Origins of self-reflective consciousness. (pp. 3-56). Nova York: Oxford University Press.

Wiggins, J. S. (1995). Interpersonal Adjective Scales: Professional manual. Odessa: Psychological Assessment Resources. 